\title{
Arthrose und rheumatoide Arthritis haben initial ähnliche Krankheitslast
}

Chua JR et al. Disease burden in osteoarthritis is similar to that of rheumatoid arthritis at initial rheumatology visit and significantly greater six months later. Arthritis and Rheumatology 2019; 71: 1276-1284

Studien deuten darauf hin, dass die Krankheitslast bei Arthrose so groß wie bei rheumatoider Arthritis (RA) ist. Die Beschwerden der Patienten werden im medizinischen Alltag oftmals mit unterschiedlichen Instrumenten abgefragt, etwa dem Western Ontario and McMaster Universities OA Index bei Arthrose und dem Multidimensional Health Assessment Questionnaire (MDHAQ) bei RA. Eine aktuelle Studie untersucht die Krankheitslast von Arthrose- und RAPatienten im Verlauf mit dem MDHAQ.

In der Studie wurden Daten von Arthroseund RA-Patienten ausgewertet, die bei der Erstvorstellung beim Rheumatologen sowie 6 Monate später den MDHAQ ausgefüllt hatten. In die Auswertung gingen Patientendaten von insgesamt 149 Arthrose- und 203 RA-Patienten aus den Jahren 2011-2017 ein. Die RA-Patienten wurden je nachdem, ob sie bereits eine DMARD (disease-modifying antirheumatic drug)-Therapie erhalten hatten, in eine von 2 Gruppen eingeteilt.

Die Beschwerden der Patienten werden im MDHAQ in Scores quantifiziert. Die Scores für körperliche Funktion, Schmerz und Gesamteinschätzung der Patienten werden im sogenannten RAPID3 Score zusammengefasst, der Werte von 0-30 annehmen kann. Die Studienautoren verglichen die RAPID3 Scores der Probanden miteinander und au- 
ßerdem die MDHAQ-Scores für Fatigue, für die selbst-angegebene Anzahl der schmerzhaften Gelenke und für eine SymptomCheckliste aus über 60 Symptomen.

Die Arthrose-Patienten in der Studie waren bei der Erstvorstellung im Mittel älter als die RA-Patienten mit und ohne vorhergegangene DMARD-Therapie (66 Jahre vs. 54 Jahre vs. 52 Jahre), hatten einen höheren BMI (33,5 vs. 27,9 vs. 30,7) und eine längere Krankheitsdauer (3,4 vs. 3,2 vs. 1 Jahre). Bei der Erstvorstellung unterschied sich der mittlere RAPID3 Score der Arthrose-Patienten nicht signifikant von dem der RA-Patienten beider Gruppen (16vs. 15,5 vs. 15,6). Interessanterweise hatten bereits $75 \%$ der RA-Patienten vor der Erstvorstellung beim Rheumatologen DMARDs erhalten.

Nach 6 Monaten sank der RAPID3 Score zwar bei allen Patientengruppen, bei RAPatienten allerdings stärker als bei den $\mathrm{Ar}$ throse-Patienten ( $-1,7$ bei Arthrose, -4.3 bei RA-Patienten mit frühere DMARD-Therapie, $-5,7$ bei RA-Patienten ohne frühere DMARD-Therapie). Die mittlere Differenz der RAPID3-Veränderungen zwischen Arthrose- und allen RA-Patienten lag bei 3,3 und war signifikant - auch wenn das höhere Alter, die längere Krankheitsdauer und der höhere BMI der Patienten berücksichtigt wurden.

Der signifikante Unterschied kommt, so erklären die Autoren, durch den Vergleich von Arthrose-Patienten und RA-Patienten ohne vorhergegangene DMARD-Therapie zustande. Der Unterschied der Scores zwischen Arthrose-Patienten und RA-Patienten mit vorhergegangener DMARD-Therapie war nicht signifikant.

FAZIT

Die Krankheitslast von Patienten mit Arthrose und rheumatoider Arthritis unterscheidet sich bei Erstvorstellung beim Rheumatologen nicht. Im Verlauf eines halben Jahres verbessert sich die Krankheitslast der RA-Patienten ohne vorhergegangene DMARD-Therapie stärker als die der Arthrose-Patienten. 